\title{
EL IMPACTO EXTERIOR DE LAS REVISTAS ESPAÑOLAS DE ECONOMÍA*
}

\author{
DANIEL A. TIRADO FABREGAT \\ JORDI PONS NOVELL
}

Universitat de Barcelona ${ }^{\mathrm{a}}$

\begin{abstract}
RESUMEN
En este trabajo se elaboran indicadores de impacto de las revistas españolas de economía teniendo en cuenta el número de ocasiones que los artículos publicados en las mismas son citados en las revistas internacionales incluidas en el ISI. La disponibilidad de estos indicadores de impacto permitirá determinar el impacto internacional de las revistas españolas, así como establecer una nueva ordenación de las mismas a partir de su impacto internacional, aspecto no considerado en los artículos publicados hasta el momento en los que se construían factores de impacto computando el número de citas que recibían las revistas españolas en otras revistas de carácter estatal. Finalmente, la comparación de estos resultados con los obtenidos por las revistas alemanas, francesas o italianas permite identificar qué factores han sido clave para la internacionalización de estas revistas de economía.
\end{abstract}

Palabras clave: bibliometría, revistas económicas, impacto

* Este trabajo ha sido realizado gracias a la financiación ofrecida por el Ministerio de Educación y Ciencia a través del proyecto de investigación EA05-0162. Los autores agradecen la colaboración de Sergio Afcha y Raimon Soler, así como las sugerencias y comentarios realizados por dos evaluadores anónimos y el editor de la revista.

a Avda. Diagonal, 690. 08034 Barcelona. E-mail: E-mail: datirado@ub.edu. E-mail: jpons@ub.edu. 


\begin{abstract}
This study looks at the international impact of leading economics journals published in Spain. For this purpose data were collected on the number of times that articles published in Spanish journals are cited in international journals on the ISI Journals lists. Constructing these kind of impact factor enables us to stablish a new ranking of Spanish leading journals arising from their international impact. This has been a neglected aspect in previous work that constructed impact factors for Spanish academic journals. Finally, the comparison of the results with those obtained for the leading Italian, French or German journals consents us to identify the basic elements which favour the internacionalization of these national economics journals.
\end{abstract}

Keywords: bibliometrics, economics journals, impact factor

JEL Classification: A11, A13

\title{
1. INTRODUCCIÓN
}

En los últimos años ha crecido el interés por la evaluación de la actividad investigadora en los distintos campos del conocimiento. Organismos públicos y privados han demandado mayor precisión en la elaboración de este tipo de información puesto que, cada vez más, se ha ligado el reconocimiento de complementos económicos para investigadores, la adjudicación de fondos a la financiación de proyectos o grupos de investigación, o el soporte a los centros públicos o privados encargados de organizar la misma, a la consecución de resultados. La cuantificación de los outputs de investigación mediante procedimientos que permitan establecer comparaciones entre investigadores, grupos o centros de investigación, en perspectiva nacional o internacional, ha pasado, por tanto, a ser un aspecto de crucial importancia.

Esta dinámica es la que ha propiciado que, al igual que en otras áreas de conocimiento, en el campo de la economía hayan aparecido los primeros trabajos encaminados a establecer ordenaciones de los centros académicos españoles en el ámbito económico a partir del número de páginas publicadas en revistas internacionales y/o nacionales de reconocido prestigio ${ }^{1}$. El enfoque en que se basan estos trabajos se fundamenta en cuantificar el número de colaboraciones en revistas científicas, de forma que sea posible elaborar una jerarquización de los centros, universidades o departamentos a partir de considerar la producción científica realizada por sus integrantes. La lógica que subyace a este procedimiento, que tiene

1 Éste sería el caso de trabajos como los de García et al. (1999a y 1999b), Pons y Tirado (1999), Bergantiños et al. (2002), Dolado et al. (2003) o Pons, Tirado y Román (2006). 
un uso muy extendido en la literatura internacional y ha sido aplicado de forma persistente en el análisis de la investigación propia de distintas ramas del conocimiento, es que la producción científica de calidad se difunde en revistas de reconocido prestigio, tanto nacionales como internacionales.

Esta opción no considera otros aspectos relevantes como los libros publicados, las monografías elaboradas o los contratos de transferencia de conocimiento a las empresas y a las Administraciones Públicas. No obstante, el enfoque bibliométrico utilizado para construir los rankings de las universidades y de las instituciones económicas que realizan investigación en economía en España es homologable al utilizado en el ámbito internacional ${ }^{2}$.

No obstante, si bien los trabajos que evalúan la producción científica en economía realizada por investigadores y/o instituciones españolas comparten metodología bibliométrica con los realizados en el ámbito internacional, se enfrentan a un problema adicional que no ha sido totalmente resuelto hasta el momento. Éste se relaciona con la forma en la que es posible realizar la valoración relativa de cada una de las páginas de un trabajo en función de la calidad del mismo. En este sentido, de forma general, en los estudios bibliométricos internacionales se ha tendido a construir y utilizar un instrumento que permite la comparación entre artículos, asignando a los mismos la calidad supuesta del medio en que han sido publicados: el factor de impacto, que es el número de ocasiones que los artículos publicados por un conjunto de publicaciones citan los artículos publicados en los años precedentes en una determinada revista.

En la literatura internacional en el ámbito de la economía se utiliza de forma preferente el indicador que, siguiendo esta filosofía, es ofrecido por el ISI. En particular, y siguiendo un ejemplo, el factor de impacto ofrecido por el ISI para el Journal of Forecasting en 2004 se obtiene, en la más extendida de sus versiones, dividiendo el número total de citas que todas las revistas del Social Science Citation Index (SSCI) efectúan en 2004 de artículos publicados en el Journal of Forecasting en 2002 y 2003 entre el número de artículos que esta revista ha publicado en estos dos años.

El problema al que se enfrentan los trabajos que tratan de ofrecer información sobre la producción científica en economía de los investigadores o centros españoles es que ésta se difunde tanto en revistas nacionales (publicadas mayoritariamente en castellano) como internacionales y que los factores de impacto normalmente empleados en la literatura internacional proceden de bases de datos que ofrecen una cobertura muy reducida, por no decir casi inexistente, de las publica-

2 A título ilustrativo, se puede destacar la utilización de metodologías similares a las empleadas en el estudio del caso español, en análisis sobre la producción científica en economía en Estados Unidos (Conroy et al. 1995, Scott y Mitias, 1996 y Dusansky y Vernon, 1998), en Europa (Kalaitzidakis et al., 2003, Combes y Linemer, 2003 y Tombazos, 2005) o en todo el mundo (Coupé, 2003). 
ciones realizadas en lenguas distintas al inglés. Así, por ejemplo, en su edición de 2004, el área de Economía del SSCI sólo incluía tres cabeceras en castellano - la española Investigaciones Económicas, la mexicana Trimestre Económico y la argentina Desarrollo Económico/Revista de Ciencias Sociales-. Este elemento dificulta que la evaluación de los curricula individuales, de grupo o de centro, pueda ser elaborada a partir de una valoración de los outputs establecida en función de unos criterios comunes para las publicaciones presentes en los listados ISI y para aquéllas que no aparecen en los mismos.

Recientemente, un pequeño grupo de trabajos ha iniciado el camino que permitirá disponer de indicadores de impacto para las revistas españolas de Economía a partir de la consideración del número de citas que éstas reciben por parte de los artículos publicados en el conjunto de revistas españolas de Economía $^{3}$. Estos trabajos, a pesar de estar sujetos a críticas y problemas relacionados con la cobertura temporal analizada o con el método empleado para la selección de las revistas analizadas, han abierto un camino que debería servir para la construcción de unos instrumentos que, finalmente, hicieran posible la comparación de los curricula de los investigadores con independencia de si publican en revistas españolas o internacionales.

El objeto de este estudio es avanzar en esta línea de trabajo, no sólo ampliando en términos de cobertura temporal o de publicaciones analizadas la información resultante de los trabajos citados anteriormente, sino planteando la construcción de indicadores que permitan establecer la comparación directa de la calidad, difusión e impacto de las publicaciones españolas en economía respecto a las revistas internacionales.

En particular, se pretende construir indicadores de impacto para las revistas españolas de economía considerando el número de ocasiones que los artículos publicados por estas revistas son citados en las incluidas en el ISI. Estos indicadores de impacto permitirán determinar el impacto real de las revistas españolas por comparación directa con las (mayoritariamente) editadas en inglés y presentes en las bases de datos internacionales. Además, como ya se ha comentado, servirá para establecer una nueva ordenación de las revistas españolas a partir de su impacto internacional, aspecto no considerado en los artículos publicados hasta el momento, en los que los factores de impacto se construían considerando únicamente contabilizando las citas recibidas por parte de otras revistas españolas. Finalmente, la comparación entre la visibilidad de las revistas españolas y la de sus homólogas de Italia, Francia y Alemania permitirá realizar una reflexión acerca de la difusión alcanzada por las cabeceras españolas, así como identificar algu-

${ }^{3}$ Éste es el caso de los trabajos elaborados por Pons y Tirado (2001), Tirado y Pons (2003), Velázquez (2003 y 2004) o EC3 (Grupo de Investigación Evaluación Científica y de la Comunicación Científica de la Universidad de Granada, http://ec3.ugr.es/in-recs). 
nas de las características propias de las revistas nacionales con mayor impacto internacional.

Con este objetivo, el resto del trabajo se organiza de la siguiente manera. En primer lugar, se presenta la metodología seguida en el estudio. En segundo lugar, se ofrece una panorámica general sobre las citas ISI recibidas por las revistas españolas de economía a lo largo de su historia. En él, además de información puramente cuantitativa, se señalan algunos puntos referentes a la calidad y características de las mismas. En tercer lugar, tras ofrecer los datos básicos relativos a las citas recibidas por los artículos publicados entre 1995 y 1999 en las revistas españolas seleccionadas en los años 1996-2004, se construyen distintos indicadores de impacto internacional de las revistas españolas de economía. En cuarto lugar, los resultados obtenidos son comparados con los derivados de los estudios que han analizado el impacto de las revistas españolas de economía a partir del número de citas recibido por los artículos publicados en las mismas en publicaciones de ámbito estatal. A continuación se presenta y analiza nueva evidencia relativa a la visibilidad de las revistas españolas de economía en comparación con la alcanzada por las principales revistas alemanas, francesas e italianas. El trabajo se cierra con un apartado dedicado a resumir las principales conclusiones.

\section{METODOLOGÍA}

La metodología propuesta para la elaboración de los indicadores de impacto es similar a la empleada por el ISI y por los trabajos ya citados que cuantifican el impacto de las revistas españolas de economía a partir de la consideración de las citas recibidas en revistas nacionales. Esta opción permite, de una parte, establecer una comparación directa con los factores de impacto ofrecidos por ISI y, por otra, con los obtenidos a partir de los trabajos realizados en España en los últimos años. Se trata, por tanto, de ofrecer una primera aproximación a los cambios que pueden registrarse en las valoraciones otorgadas a las diferentes revistas españolas en función del ámbito de difusión considerado para sus trabajos (revistas internacionales versus revistas españolas).

Así, la metodología propuesta para la construcción de estos indicadores no difiere en gran medida de la empleada por el ISI y permite la construcción de indicadores de impacto perfectamente comparables (por construcción) con los ofrecidos por el ISI. En particular, la utilización de esta metodología supone la realización inicial de las siguientes tareas:

a) Selección de las revistas españolas objeto del estudio.

b) Selección de las revistas ISI a considerar. 
Por lo que respecta a la selección de la muestra de revistas españolas se ha optado por tomar como referencia los trabajos que han tratado de analizar el impacto de las mismas en publicaciones de ámbito estatal. En particular, se han seleccionado para su estudio las 30 primeras revistas españolas ordenadas según su factor de impacto básico en el trabajo realizado sobre este aspecto por Velázquez (2003). La opción implica incluir en la muestra un número muy amplio de publicaciones. De hecho, como se verá, significa la inclusión de cabeceras que no han recibido ninguna cita de las revistas del ISI a lo largo de su historia. En el cuadro 1 se presenta la relación de títulos considerados.

En segundo lugar se ha determinado el grupo de publicaciones ISI sobre las que establecer el análisis. En este sentido, se ha optado por no restringir el conjunto de información a una muestra de las mismas y considerar todas las citas recibidas por los artículos publicados en las revistas españolas en cualquier publicación presente en las bases de datos ISI. La opción permitirá, a posteriori, establecer acotaciones en función del ámbito temporal de las citas o del impacto internacional de las revistas consideradas.

Partiendo de estas consideraciones se ha elaborado la información referente a las citas recibidas por los artículos publicados en las 30 revistas españolas con mayor factor de impacto nacional según Velázquez (2003) a lo largo de su historia. La presentación de esta información es el objeto del siguiente de los apartados.

CUADRO 1

REVISTAS ESPAÑOLAS ANALIZADAS

\begin{tabular}{l|l} 
Moneda y Crédito & Cuadernos de Información Económica \\
Investigaciones Económicas & Boletín de Estudios Económicos \\
Papeles de Economía Española & Revista Técnica del ICAJCE \\
Revista de Economía Aplicada & Revista Europea de Dirección \\
Revista Española de Financiación y & y Economía de la Empresa \\
Contabilidad & Economía Industrial \\
Revista Española de Economía (Spanish & Situación \\
Economic Review) (*) & Ekonomiaz \\
Análisis Financiero & Revista Asturiana de Economía \\
Información Comercial Española & Boletín AECA \\
Hacienda Pública Española & Harvard Deusto Business Review \\
Cuadernos Económicos del ICE & Economistas \\
Perspectivas del Sistema Financiero & Revista de Historia Económica \\
Técnica Contable & Revista de Historia Industrial \\
Boletín Económico del Banco de España & Auditoria Pública \\
Partida Doble & Revista del Instituto de Estudios \\
Actualidad Financiera & Económicos
\end{tabular}

Nota: (*) A partir de 1999 esta revista pasa a publicarse íntegramente en inglés. 


\section{LAS CITAS INTERNACIONALES DE LAS REVISTAS ESPAÑOLAS DE ECONOMÍA}

En esta sección se presenta información referente a las citas ISI recibidas por las revistas españolas de economía a lo largo de su historia. En este sentido, el primer dato reseñable es que de las 30 revistas españolas analizadas, un total de cuatro nunca ha recibido citas por parte de artículos publicados en revistas presentes en bases de datos ISI.

El cuadro 2 contempla un resumen de la información existente en las bases de datos ISI acerca de las citas recibidas por las 26 revistas españolas de economía que han recibido como mínimo una cita a lo largo de toda su historia. En particular se presentan ocho columnas que recogen la siguiente información. Las dos primeras ofrecen la relación de revistas analizadas ordenadas según el total de citas recibidas a lo largo de su historia. La columna 3 presenta el número de citas recibidas por cada revista.

Las columnas 4 a 8 ofrecen información adicional sobre las características de las citas, lo que nos permitirá reflexionar sobre la calidad de las mismas. En este sentido, la columna 4 («Citas por citante») indica el número de veces que como media ha sido citado el artículo ISI citante de un artículo publicado en una revista española, es decir, el número medio de citas recibidas por los artículos internacionales que citan trabajos publicados en revistas españolas. La columna 5 («Autocitas») recoge el porcentaje de citas internacionales que han sido realizadas por los propios autores del artículo publicado en la revista española. En particular, en caso de existencia de más de un autor en los artículos analizados, se ha considerado como autocita toda cita en la que aparece como autor del artículo internacional al menos uno de los autores del artículo español citado.

Por otra parte, la columna 6 («\% Filiación española») aporta información referente a la filiación profesional de los autores. En particular, se ofrece el dato relativo al porcentaje de autores citantes de artículos publicados en revistas españolas que tienen al menos una filiación profesional en una institución de investigación española. En el caso de artículos publicados por más de un investigador se ha considerado suficiente el hecho de que uno de ellos declarara su pertenencia a un centro de investigación español.

Las columnas 7 y 8 presentan información relativa a las revistas ISI en las que aparecen las citas de artículos publicados en revistas españolas. En particular, la columna 7 ( $\%$ No FI ISI») nos indica el porcentaje de citas realizadas en revistas presentes en bases de datos ISI pero que carecen de factor de impacto para los años 2000-2004. La columna 8 («FI ISI, 2000-04»), por el contrario, recoge el factor de impacto medio calculado por ISI para las revistas citantes durante los años 2000-2004. 
CUADRO 2

CITAS ISI REVISTAS ESPAÑOLAS DE ECONOMÍA EN TODA SU HISTORIA

\begin{tabular}{|c|c|c|c|c|c|c|}
\hline & $\begin{array}{l}\text { Total } \\
\text { citas }\end{array}$ & $\begin{array}{l}\text { Citas } \\
\text { por } \\
\text { citante }\end{array}$ & Autocitas & $\begin{array}{c}\% \\
\text { Filiación } \\
\text { Española }\end{array}$ & $\begin{array}{l}\% \text { No } \\
\text { FI ISI }\end{array}$ & $\begin{array}{c}\text { FI ISI } \\
(2000-04)\end{array}$ \\
\hline 1. Moneda y Crédito............... & 308 & 2,07 & 6,60 & 52,29 & 42,86 & 0,68 \\
\hline 2. Investigaciones & & & & & & \\
\hline Económicas ...... & 304 & 2,43 & 13,49 & 70,39 & 0,99 & 0,75 \\
\hline $\begin{array}{l}\text { 3. Papeles de Economía } \\
\text { Española .......................... }\end{array}$ & 293 & 2,37 & 6,84 & 61,77 & 8.19 & 0.71 \\
\hline 4. Revista de Historia & & & & & & \\
\hline Económica ............... & 263 & 1,81 & 10,77 & 47,53 & 48,67 & 0,58 \\
\hline 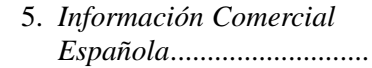 & 191 & 1,39 & 10,75 & 69,49 & 22,51 & 0,46 \\
\hline $\begin{array}{l}\text { 6. Revista Española } \\
\text { de Economía (SER).......... } \\
\text { 7. Hacienda Pública }\end{array}$ & 164 & 3,04 & 21,34 & 55,19 & 1,83 & 0,60 \\
\hline Española.................. & 155 & 1,57 & 16,13 & 68,15 & 45,16 & 0,66 \\
\hline 8. Economía Industrial......... & 139 & 1,38 & 7,19 & 74,82 & 7,91 & 0,59 \\
\hline $\begin{array}{l}\text { 9. Revista de Economía } \\
\text { Aplicada ............................ }\end{array}$ & 86 & 2,28 & 19,77 & 90,59 & 2,33 & 0,54 \\
\hline 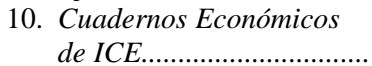 & 49 & 0,90 & 16,33 & 81,58 & 50,00 & 1,03 \\
\hline 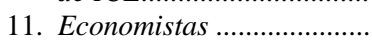 & 30 & 1,77 & 3,33 & 60,00 & 6,67 & 0,47 \\
\hline 12. Harvard Deusto................. & 30 & 1,53 & 16,67 & 63,33 & 0,00 & 0,34 \\
\hline $\begin{array}{l}\text { 13. Revista de Historia } \\
\text { Industrial ........................... } \\
\text { 14. Revista del Instituto }\end{array}$ & 29 & 0,31 & 17,24 & 96,55 & 34,48 & 0,43 \\
\hline $\begin{array}{l}\text { de Estudios Económicos... } \\
\text { 15. Revista Asturiana }\end{array}$ & 17 & 17,24 & 17,65 & 41,18 & 11,76 & 0,63 \\
\hline de Economía.............. & 16 & 2,50 & 18,75 & 80,00 & 0,00 & 0,77 \\
\hline 16. Ekonomiaz............... & 16 & 2,69 & 18,75 & 75,00 & 31,25 & 0,82 \\
\hline $\begin{array}{l}\text { 17. Revista Europea } \\
\text { de Dirección ....................... }\end{array}$ & 14 & 0,64 & 28,57 & 100,00 & 0,00 & 0,41 \\
\hline 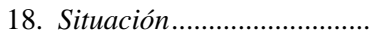 & 11 & 1,73 & 9,09 & 100,00 & 9,09 & 0,54 \\
\hline $\begin{array}{l}\text { 19. Perspectivas Sistema } \\
\text { Financiero .......................... }\end{array}$ & 4 & 6,00 & 0,00 & 75,00 & 0,00 & 0,52 \\
\hline $\begin{array}{l}\text { 20. Auditoría Pública .................. } \\
\text { 21. Boletín Económico }\end{array}$ & 3 & 0,33 & 0,00 & 100,00 & 0,00 & 0,60 \\
\hline del Banco de España......... & 3 & 1,00 & 0,00 & 33,33 & 0,00 & 0,67 \\
\hline 22. Partida Doble ................... & 2 & 0,00 & 0,00 & 100,00 & 0,00 & 0,48 \\
\hline 23. Actualidad Financiera...... & 2 & 0,00 & 50,00 & 50,00 & 50,00 & 0,59 \\
\hline 24. Técnica Contable............... & 2 & 0,00 & 0,00 & 100,00 & 0,00 & 0,41 \\
\hline $\begin{array}{l}\text { 25. Análisis Financiero ............ } \\
\text { 26. Revista Española } \\
\text { de Financiación }\end{array}$ & 1 & 0,00 & 0,00 & 0,00 & 0,00 & 0,22 \\
\hline y Contabilidad...... & 1 & 0,00 & 100,00 & 100,00 & 0,00 & 0,61 \\
\hline
\end{tabular}

Fuente: Elaboración propia. 
Del análisis de los datos históricos de citas a los artículos publicados por las revistas españolas de economía por parte de los artículos publicados en revistas presentes en las bases de datos de ISI se desprende que el impacto internacional de un conjunto importante de revistas españolas es puramente testimonial. Baste señalar que más de la mitad de las 30 revistas analizadas ha recibido menos de 20 citas a lo largo de su historia. No obstante, para cerca de un tercio de la muestra esta presencia no debe calificarse de irrelevante, ya que sus artículos han sido citados en más de un centenar de ocasiones. En este sentido, destacan revistas con una larga y estable trayectoria histórica como Moneda y Crédito, Investigaciones Económicas, Papeles de Economía Española y Revista de Historia Económica que, con más de 200 citas en su haber, encabezan por este orden el ranking nacional.

Por término medio, los artículos publicados en revistas internacionales que citan trabajos publicados en revistas españolas han recibido aproximadamente dos citas. Este valor se puede interpretar como un indicio que el impacto de estos artículos no es en absoluto despreciable. Se observa, además, como la presencia de autocitas es bastante limitada, puesto que supone alrededor del 20 por cien de las citas recibidas. Sin embargo, las citas recibidas por los artículos publicados en revistas españolas es muy dependiente de la presencia de autores con filiación en centros de investigación españoles en las publicaciones ISI. Como media, cerca del 70 por cien de las citas analizadas son realizadas en trabajos en los que alguno de los autores tiene esta filiación.

Finalmente, cabe señalar que el porcentaje de citas realizadas en revistas con factor de impacto 2000-2004 en las bases de datos ISI es muy irregular y, aparentemente, se relaciona con el campo de especialización de la revista citada. Así, publicaciones ligadas a áreas como la Hacienda Pública o la Historia Económica tiene un porcentaje de citas en revistas sin factor de impacto superior a aquéllas que podríamos considerar como más generalistas, o propias de campos específicos como el de Fundamentos del Análisis Económico. Además, el factor de impacto medio de las revistas ISI en las que aparecen citados artículos publicados en revistas españolas de economía debe considerarse como medio-bajo. Prácticamente en todos los casos se sitúa por debajo de la unidad.

\section{CITAS ISI Y FACTORES DE IMPACTO DE LAS REVISTAS ESPAÑOLAS DE ECONOMÍA PUBLICADAS ENTRE 1995 Y 1999}

En este apartado se ofrece un conjunto de información similar al detallado en el apartado previo pero acotando el periodo de estudio a los años 1995-1999. Es decir, se analiza el número de citas recibidas por cada artículo publicado en los años 1995-1999 desde su publicación hasta 2004. En este sentido, la selección de un quinquenio elimina el sesgo en favor de las revistas con más tradición que supone la consideración del total de la información histórica. 
CUADRO 3

CITAS ISI RECIBIDAS POR LOS ARTÍCULOS PUBLICADOS

ENTRE 1995 Y 1999 EN EL QUINQUENIO POSTERIOR A SU PUBLICACIÓN

\begin{tabular}{|c|c|c|c|c|c|c|}
\hline & $\begin{array}{l}\text { Total } \\
\text { citas }\end{array}$ & $\begin{array}{l}\text { Citas } \\
\text { por } \\
\text { citante }\end{array}$ & Autocitas & $\begin{array}{c}\% \\
\text { Filiación } \\
\text { Española }\end{array}$ & $\begin{array}{l}\% \text { No } \\
\text { FI ISI }\end{array}$ & $\begin{array}{c}\text { FI ISI } \\
(2000-04)\end{array}$ \\
\hline $\begin{array}{l}\text { Investigaciones Económicas. } \\
\text { Papeles de Economía }\end{array}$ & 110 & 1,48 & 19,82 & 70,39 & 0,99 & 0,66 \\
\hline 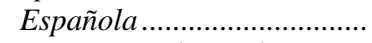 & 83 & 1,37 & 4,82 & 84,34 & 3,61 & 0,61 \\
\hline $\begin{array}{l}\text { Economía Industrial ................ } \\
\text { Revista Española }\end{array}$ & 78 & 0,95 & 5,13 & 74,14 & 0,00 & 0,41 \\
\hline $\begin{array}{l}\text { de Economía }(\text { SER) ............. } \\
\text { Información Comercial }\end{array}$ & 68 & 3,16 & 16,18 & 48,53 & 1,47 & 0,65 \\
\hline $\begin{array}{l}\text { Española ............................. } \\
\text { Revista de Historia }\end{array}$ & 48 & 1,29 & 16,67 & 83,33 & 2,08 & 0,41 \\
\hline $\begin{array}{l}\text { Económica ........................... } \\
\text { Revista de Economía }\end{array}$ & 45 & 1,27 & 13,33 & 57,78 & 31,11 & 0,62 \\
\hline Aplicada ............................ & 39 & 1,56 & 7,69 & 84,62 & 5,13 & 0,58 \\
\hline Moneda y Crédito ................... & 31 & 1,35 & 6,45 & 64,52 & 3,23 & 0,57 \\
\hline $\begin{array}{l}\text { Hacienda Pública Española. } \\
\text { Revista Asturiana }\end{array}$ & 20 & 0,65 & 25,00 & 100,00 & 15,00 & 0,39 \\
\hline de Economía ........................... & 14 & 2,86 & 14,29 & 71,43 & 0,00 & 0,80 \\
\hline $\begin{array}{l}\text { Economistas......................................... } \\
\text { Revista Europea }\end{array}$ & 11 & 2,27 & 9,09 & 77,78 & 0,00 & 0,56 \\
\hline de Dirección ....................... & 11 & 0,73 & 36,36 & 100,00 & 0,00 & 0,37 \\
\hline Revista de Historia Industrial & 10 & 0,50 & 20,00 & 90,00 & 20,00 & 0,47 \\
\hline Harvard Deusto ..................... & 9 & 0,56 & 11,11 & 66,67 & 0,00 & 0,53 \\
\hline $\begin{array}{l}\text { Cuadernos Económicos } \\
\text { de ICE } . . . . . . . . . . . . . . . . . . . . . . . . . . . .\end{array}$ & 8 & 0,38 & 0,00 & 87,50 & 0,00 & 0,51 \\
\hline $\begin{array}{l}\text { Ekonomiaz ...................................... } \\
\text { Revista del Instituto }\end{array}$ & 7 & 2,57 & 14,29 & 85,71 & 28,57 & 0,67 \\
\hline de Estudios Económicos..... & 4 & 0,00 & 25,00 & 75,00 & 0,00 & 0,49 \\
\hline Auditoría Pública ................. & 3 & 0,33 & 0,00 & 100,00 & 0,00 & 0,60 \\
\hline Situación ............................... & 2 & 0,50 & 0,00 & 100,00 & 0,00 & 0,19 \\
\hline 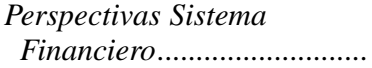 & 2 & 1,00 & 0,00 & 50,00 & 0,00 & 0,32 \\
\hline Partida Doble ....................... & 2 & 0,00 & 0,00 & 100,00 & 0,00 & 0,48 \\
\hline Actualidad Financiera .......... & 1 & 0,00 & 100,00 & 100,00 & 0,00 & 0,59 \\
\hline $\begin{array}{l}\text { Análisis Financiero................ } \\
\text { Boletín Económico }\end{array}$ & 1 & 0,00 & 0,00 & 0,00 & 0,00 & 0,21 \\
\hline del Banco de España ........... & 0 & n.d. & n.d. & n.d. & n.d. & n.d. \\
\hline Técnica Contable .................... & 0 & n.d. & n.d. & n.d. & n.d. & n.d. \\
\hline $\begin{array}{l}\text { Revista Española } \\
\text { de Financiación } \\
\text { y Contabilidad ..................... }\end{array}$ & 0 & n.d. & n.d. & n.d. & n.d. & n.d. \\
\hline
\end{tabular}

Fuente: Elaboración propia.

Nota: No se han contemplado las cuatro revistas que en el cuadro 2 aparecían con cero citas. 
Por otra parte, la selección del quinquenio 1995-1999 permite el estudio de unos años en los que han permanecido en activo todas las cabeceras que aparecen como más relevantes en los estudios de impacto nacional de las publicaciones en economía. Así, por ejemplo, de un artículo publicado en 1996 se contabilizan las citas recibidas desde esa fecha hasta 2004. Además, se ha seleccionado 1999 como el último de los años considerados con el objeto de que puedan ser analizadas las citas recibidas por los artículos publicados durante ese año a lo largo, al menos, de todo un quinquenio posterior, 2000-2004. Esta información se contempla en el cuadro 3. A continuación se realiza una breve descripción de los elementos más destacados de la misma.

Cuando se acota el conjunto de información a un solo quinquenio, se pone de manifiesto que el impacto internacional de la mayoría de las revistas españolas de economía es puramente testimonial. Baste señalar que para más de la mitad de las 30 revistas analizadas los artículos publicados durante los años 1995-1999 han recibido menos de 10 citas. Así, 14 de ellas han recibido menos de cinco citas. Por otra parte, los artículos publicados por siete de ellas no han sido citados en ninguna ocasión en revistas internacionales. De nuevo, sin embargo, para un grupo de publicaciones la relevancia internacional no es despreciable. Cuatro publicaciones han recibido más de 50 citas. Ocho más de 30. Trece, diez o más. Los comentarios que se realizan a continuación quedan acotados a este conjunto de cabeceras.

Entre las revistas más citadas, Investigaciones Económicas, Papeles de Economía Española, Economía Industrial y Revista Española de Economía (SER), con más de 50 citas en su haber, encabezan por este orden el ranking nacional. Respecto a la clasificación histórica pierden su posición entre las cuatro primeras Moneda y Crédito y Revista de Historia Económica, que son reemplazadas por Economía Industrial y Revista Española de Economía (SER).

Por lo que respecta al número de veces que han sido citados los artículos citantes de los trabajos publicados en las revistas españolas, como media, éste sigue siendo del orden de dos, aunque ahora la dispersión parece ser un poco mayor que en el caso del análisis histórico. En este sentido, destaca el elevado valor que alcanza este indicador en el caso de Revista Española de Economía (SER): los artículos ISI citantes de los artículos publicados en esta revista han sido citados, a su vez, en 3,16 ocasiones. La parte baja de esta clasificación la encabezaría Revista de Historia Industrial, que registra un valor de 0,5 para este indicador.

Las citas recibidas por los artículos publicados en revistas españolas siguen siendo muy dependientes de la presencia de autores con filiación en centros de investigación españoles en las publicaciones ISI. Como media, más del 70 por cien de las citas analizadas son realizadas en trabajos en los que alguno de los autores tiene esta filiación. En este caso destacan también Hacienda Pública Española y Revista Europea de Dirección y Economía de la Empresa. El 100 por 
cien de las citas recibidas por artículos publicados en estas dos revistas han sido realizados por autores con filiación española. Por el contrario, en el caso de Revista Española de Economía el indicador registra un valor inferior al 50 por cien.

El porcentaje de citas realizadas en revistas que carecen de factor de impacto 2000-2004 en las bases de datos ISI es, como media, despreciable. Sólo cobra alguna relevancia en el caso de revistas con marcada especialización temática como Revista de Historia Económica y Revista de Historia Industrial en el área de Historia e Instituciones Económicas o Hacienda Pública Española en el de la Hacienda Pública. En estos casos el porcentaje se sitúa alrededor del 30 por cien.

Finalmente, cabe destacar que el factor de impacto medio de las revistas ISI en las que aparecen citados artículos publicados en revistas españolas de economía debe considerarse como medio-bajo. En todos los casos se sitúa claramente por debajo de la unidad. En cualquier caso, cabe destacar que tres de las cinco revistas que reciben un mayor volumen de citas (Investigaciones Económicas, Papeles de Economía Española y Revista Española de Economía) también se sitúan entre las cinco revistas que encabezan el ranking de este indicador de calidad de las citas recibidas.

No obstante, como se ha señalado, habitualmente se construyen indicadores de impacto de las distintas publicaciones siguiendo una metodología que propone, en primer lugar, la contabilización del número de citas recibidas por los artículos publicados por una revista durante un periodo de tiempo a lo largo de un conjunto acotado de años posteriores a su publicación. En segundo lugar, este indicador bruto es corregido considerando el total de artículos publicados por cada revista en el periodo de tiempo analizado, o por el total de páginas estandarizadas o cualquier otro corrector que permita realizar una aproximación razonable al volumen de producción científica editada por cada revista en el lapso considerado.

Siendo esta la filosofía básica de la construcción de indicadores de impacto, en la literatura internacional en el ámbito de la economía se utiliza de forma preferente el indicador que, siguiendo esta filosofía, es ofrecido por el ISI en su Journal of Citations Report. Recordemos, siguiendo un ejemplo ya citado anteriormente, que el factor de impacto ofrecido por el ISI para el Journal of Forecasting en 2004 se obtiene, en la más extendida de sus versiones, dividiendo el número total de citas que todas las revistas del Social Science Citation Index efectúan en 2004 de artículos publicados en el Journal of Forecasting en 2002 y 2003 entre el número de artículos que esta revista ha publicado en estos dos años.

No obstante, si se considera el reducido número de citas que reciben los artículos publicados por las revistas españolas de economía en el ámbito internacional, la construcción de este tipo de indicador podría generar fuertes sesgos en función de los años seleccionados. Por ello se ha preferido construir indicadores de 
impacto que, siguiendo la filosofía de los construidos por el ISI, mantuvieran las especificidades necesarias para el estudio de los casos nacionales. En particular, se propone la construcción de un factor de impacto que recoja el total de citas recibidas por los artículos publicados en las revistas españolas en los años 1995-1999 desde su publicación hasta 2004. Además, como ponderador del volumen agregado de citas se ha utilizado, al igual que en la versión más extendida del factor de impacto ofrecido por ISI, el número total de artículos publicados por las revistas en los años objeto de análisis.

Los resultados de la realización de este ejercicio son los que se presentan en el cuadro 4, que a continuación se pasa a comentar. En este cuadro, «FI» es el cociente entre el número de citas recibidas y los artículos publicados en el periodo objeto de estudio, mientras que «Índice FI» recoge la misma información pero asignando a la primera revista de la clasificación el valor 100 .

\section{CUADRO 4}

FACTOR DE IMPACTO 1995-1999 PONDERADO POR EL NÚMERO DE ARTÍCULOS PUBLICADOS

\begin{tabular}{|c|c|c|}
\hline & FI & Índice FI \\
\hline 1. Revista Española de Economía (SER) & 1,13 & 100,0 \\
\hline 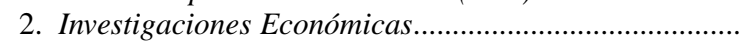 & 1,05 & 92,7 \\
\hline 3. Moneda y Crédito............ & 0,56 & 49,9 \\
\hline 4. Revista de Historia Económica..... & 0,38 & 33,5 \\
\hline 5. Revista de Economía Aplicada.. & 0,36 & 31,7 \\
\hline 6. Papeles de Economía Española .. & 0,21 & 18,4 \\
\hline 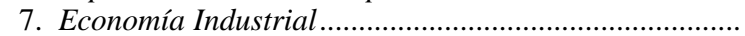 & 0,18 & 15,7 \\
\hline 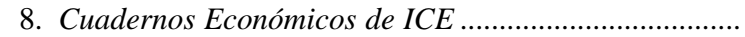 & 0,17 & 14,8 \\
\hline 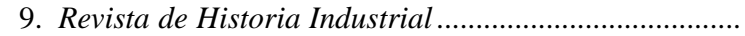 & 0,14 & 12,0 \\
\hline 10. Revista Asturiana de Economía ... & 0,11 & 9,3 \\
\hline 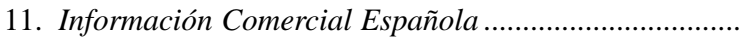 & 0,09 & 7,9 \\
\hline 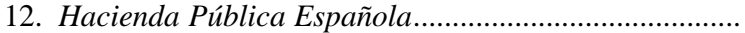 & 0,07 & 6,1 \\
\hline 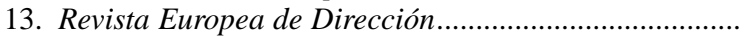 & 0,05 & 4,8 \\
\hline 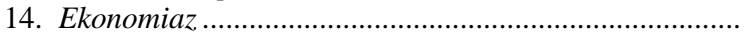 & 0,04 & 3,5 \\
\hline 15. Harvard Deusto & 0,03 & 2,7 \\
\hline 16. Economistas & 0,02 & 1,7 \\
\hline 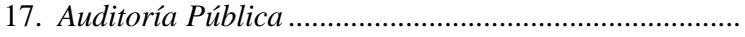 & 0,02 & 1,6 \\
\hline 18. Revista del Instituto de Estudios Económicos ............... & 0,02 & 1,4 \\
\hline 19. Situación & 0,01 & 1,2 \\
\hline 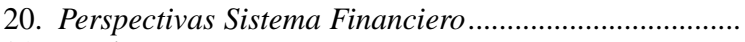 & 0,01 & 1,2 \\
\hline 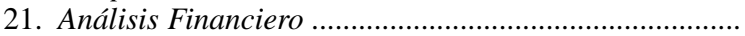 & 0,01 & 0,8 \\
\hline 22. Partida Doble & 0,00 & 0,4 \\
\hline 23. Actualidad Financiera & 0,00 & 0,1 \\
\hline
\end{tabular}

Fuente: Elaboración propia.

Nota: No se han contemplado las siete revistas que en el cuadro 2 o en el 3 aparecían con cero citas. 
En primer lugar, cabe destacar el bajo factor de impacto asociado a la mayor parte de revistas españolas de economía. En particular, sólo Revista Española de Economía (Spanish Economic Review) e Investigaciones Económicas reciben un número de citas superior al número de artículos publicado en los años 1995$1999^{4}$. En la mayor parte de casos, el número de artículos publicados es más de diez veces superior al de citas. Para ocho de las 23 revistas analizadas, el factor de impacto obtenido indica que sólo uno de cada cincuenta artículos publicados ha recibido alguna cita internacional en el periodo de referencia.

Atendiendo a esta evidencia, se concluye que el análisis del impacto internacional de las revistas españolas debería centrarse en dos aspectos fundamentales. El estudio del orden de relevancia de las publicaciones españolas en cuanto a su impacto internacional a través de la construcción de de diferentes índices de impacto, y la construcción de indicadores de impacto comparables con aquéllos propios de las revistas nacionales de economía de países en los que la lengua de publicación básica no sea el inglés. Este tipo de estudio es el que serviría para dilucidar si los bajos factores de impacto calculados para las revistas españolas son comparables o no a los obtenidos para otras publicaciones homólogas francesas, italianas o alemanas. El primero de estos aspectos pasa a ser el objeto de atención en las páginas que siguen. El segundo será materia de análisis en futuros trabajos.

En este sentido, la última de las columnas del cuadro 4 ofrece índices con base 100 a la revista de mayor difusión de las revistas españolas de economía que obtienen citas en el periodo considerado. A partir de su análisis se puede señalar que dos revistas destacan claramente por encima de la media en cuanto a su impacto internacional: Revista Española de Economía (Spanish Economic Review) e Investigaciones Económicas. Además, un grupo de tres cabeceras se situaría, aproximadamente, en el segundo tercio de la distribución. En él se encuadrarían Moneda y Crédito, Revista de Historia Económica y Revista de Economía Aplicada. Las cinco siguientes cabeceras constituirían un grupo con índices de impacto entre 10 y 20 . Finalmente, el resto de revistas ofrecen índices inferiores a 10 , con 10 revistas obteniendo índices inferiores a 5 .

A partir de estos resultados se desprende la existencia de marcadas diferencias en cuanto al impacto internacional de los artículos publicados en revistas españolas. Así, por comparación con los resultados ofrecidos en el cuadro 3 sobre volumen bruto de citas recibidas, cabe destacar cómo tres de las cinco cabeceras que recibían un mayor volumen de citas brutas no se sitúan entre las cinco primeras cuando se realiza una ponderación en función del total de artículos publicados.

${ }^{4}$ No puede olvidarse que estas dos revistas publican una parte de sus artículos en inglés (y en el caso de Revista Española de Economía a partir de 1999 se publica íntegramente en este idioma). Esta circunstancia puede influir en los resultados obtenidos. 
Papeles de Economía Española, Economía Industrial e Información Comercial Española abandonan este grupo y son reemplazadas por Moneda y Crédito, Revista de Historia Económica y Revista de Economía Aplicada.

Con el objeto de comprobar si los resultados obtenidos están ligados al factor de corrección empleado (número de artículos publicados), en el cuadro 5 se ofrecen índices de impacto de las diez primeras revistas que, en este caso, han sido calculados mediante la ponderación de las citas brutas por el total de páginas estandarizadas publicadas, considerando el número de caracteres por página calculados en Pons y Tirado (1999).

Se comprueba cómo en el caso de utilizar este factor de corrección, el ranking de revistas sólo sufre una variación respecto al que se establecía cuando el elemento de corrección empleado era el número de artículos. Ahora las dos primeras revistas (Investigaciones Económicas y Revista Española de Economía) intercambian sus posiciones. El resto de la clasificación se mantiene estable.

Por lo que respecta a los nuevos valores del índice, cabe destacar que permiten mantener una clasificación por grupos de revistas similar a la descrita en los párrafos previos. Es decir, las dos revistas citadas encabezan la clasificación con índices situados en el tercio superior de la distribución. Tres estarían en torno al segundo tercio de la misma (cabría matizar que, estrictamente, ahora Revista de Economía Aplicada quedaría cinco puntos porcentuales por debajo de este lumbral). Finalmente las cinco últimas cabeceras consideradas se situarían en el último de los tercios de la distribución, pero con valores del índice superiores a diez. En líneas generales, por tanto, se puede considerar que los resultados obtenidos se mantienen con independencia del factor de corrección aplicado en el cálculo del factor impacto.

CUADRO 5

ÍNDICE DE IMPACTO CORREGIDO POR EL NÚMERO DE PÁGINAS ESTANDARIZADAS PUBLICADAS

\begin{tabular}{|c|c|}
\hline & Índice de impacto \\
\hline 1. Investigaciones Económicas .... & 100,0 \\
\hline 2. Revista Española de Economía (SER).. & 69,9 \\
\hline 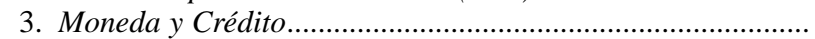 & 42,9 \\
\hline 4. Revista de Historia Económica... & 40,3 \\
\hline 5. Revista de Economía Aplicada ... & 28,1 \\
\hline 6. Papeles de Economía Española.... & 19,2 \\
\hline 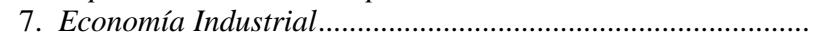 & 17,4 \\
\hline 8. Cuadernos Económicos de ICE... & 14,0 \\
\hline 9. Revista de Historia Industrial... & 12,2 \\
\hline 10. Revista Asturiana de Economía... & 11,8 \\
\hline
\end{tabular}

Fuente: Pons y Tirado (1999) y elaboración propia. 


\section{4. ÍNDICES DE IMPACTO INTERNACIONAL VS. INTERIOR: UN ANÁLISIS COMPARADO}

En este apartado se ofrece una primera comparación entre el impacto internacional de las revistas españolas de economía y su impacto interior. Este tipo de análisis permitirá establecer la existencia o no de diferencias relevantes en los ámbitos de difusión de las revistas analizadas. Con este objeto se comparan los resultados obtenidos en el análisis bibliométrico realizado en esta investigación con los obtenidos con otros de características similares pero que centran su atención en el estudio del impacto interior de las revistas españolas. En particular, en el cuadro que se ofrece a continuación y en los cometarios que siguen se ha utilizado como elemento de comparación los resultados obtenidos en Velázquez (2003).

CUADRO 6

COMPARATIVA ÍNDICES DE IMPACTO INTERNACIONAL VS. INTERIOR

\begin{tabular}{|c|c|c|c|}
\hline & \multirow{2}{*}{$\begin{array}{c}\text { Índice FI } \\
\text { internacional }\end{array}$} & \multicolumn{2}{|c|}{ Velázquez (2003) } \\
\hline & & Índice nacional & Ranking \\
\hline 1. Revista Española de Economía $(S E R)$....... & 100,0 & 57,5 & 5 \\
\hline 2. Investigaciones Económicas ...................... & 92,7 & 89,6 & 2 \\
\hline 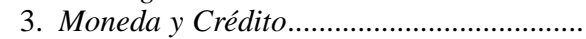 & 49,9 & 100,0 & 1 \\
\hline 4. Revista de Historia Económica........ & 33,5 & 23,3 & 15 \\
\hline 5. Revista de Economía Aplicada ......... & 31,7 & 69,4 & 3 \\
\hline 6. Papeles de Economía Española.... & 18,4 & 66,8 & 4 \\
\hline 7. Economía Industrial........................ & 15,7 & 24,9 & 14 \\
\hline 8. Cuadernos Económicos de ICE... & 14,8 & 33,0 & 9 \\
\hline 9. Revista de Historia Industrial.... & 12,0 & 13,2 & 21 \\
\hline 10. Revista Asturiana de Economía... & 9,3 & 17,6 & 18 \\
\hline 11. Información Comercial Española... & 7,9 & 39,3 & 7 \\
\hline 12. Hacienda Pública Española ............. & 6,1 & 45,9 & 6 \\
\hline 13. Revista Europea de Dirección ......... & 4,8 & 31,3 & 10 \\
\hline 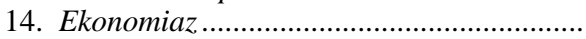 & 3,5 & 20,1 & 16 \\
\hline 15. Harvard Deusto.............................. & 2,7 & 14,6 & 20 \\
\hline 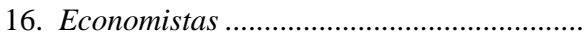 & 1,7 & 15,5 & 19 \\
\hline 17. Auditoría Pública ......................... & 1,6 & 10,7 & 22 \\
\hline 18. Revista del Instituto de Estudios & & & \\
\hline 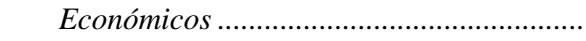 & 1,4 & 10,5 & 23 \\
\hline 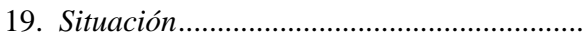 & 1,2 & 17,7 & 17 \\
\hline 20. Perspectivas Sistema Financiero....... & 1,2 & 30,9 & 11 \\
\hline 21. Análisis Financiero ...................... & 0,8 & 39,1 & 8 \\
\hline 22. Partida Doble ..................... & 0,4 & 28,6 & 12 \\
\hline 23. Actualidad Financiera.... & 0,1 & 28,5 & 13 \\
\hline
\end{tabular}

Fuente: Velázquez (2003) y elaboración propia. 
Así, en el cuadro 6 en las columnas 1 a 3 se replican los resultados obtenidos en este estudio y, a continuación, en las columnas 4 y 5 se ofrecen los resultado obtenidos para las mismas revistas en el trabajo realizado por Velázquez (2003). En particular, se presenta el índice básico de impacto calculado por este autor para estas revistas y el orden de clasificación que cada una de ellas alcanza en su trabajo. Por ejemplo, en este trabajo Revista Española de Economía alcanza el primer puesto con un índice de impacto 100, mientras que en el trabajo de Velázquez esta publicación obtiene un índice de impacto de 57,5 que le permite situarse en el puesto núm. 5 de la clasificación.

Del análisis de ambos resultados se desprende que algunas publicaciones mantienen puestos privilegiados, por lo que respecta a su impacto, con independencia de cuál sea el observatorio utilizado (mercado interior o internacional). Éste sería el caso de cabeceras como Revista Española de Economía, Investigaciones Económicas, Moneda y Crédito, Revista de Economía Aplicada o Papeles de Economía Española. Éstas ocupan las primeras posiciones del ranking en cualquiera de los casos.

Pero también se observa la existencia de revistas en las que las diferencias en el impacto asociado a la publicación varían de forma manifiesta según sea el ámbito de difusión analizado. Por ejemplo, revistas de marcada especialización en el área de Historia e Instituciones Económicas como Revista de Historia Económica y Revista de Historia Industrial, mejoran de forma manifiesta su posición en caso de considerar el impacto internacional y no el interior. La primera de ellas pasa de ocupar el puesto 15 al 5. La segunda del 21 al 9.

Sin embargo, revistas asociadas a otras especializaciones temáticas, como la Contabilidad y la Economía Financiera, pierden posiciones en caso de centrar la atención en su impacto exterior. Así, Análisis Financiero pasa del lugar 8 del ranking nacional al 21. Revista Española de Financiación y Contabilidad, que ocupaba el puesto núm. 3 en el trabajo de Velázquez o Técnica Contable, que ocupaba el 10, no aparecen en el cuadro 6 , al no haber recibido ninguna cita ISI en el periodo objeto de análisis.

Finalmente, cabe destacar cómo revistas de amplio impacto en el mercado interior como Hacienda Pública Española (puesto 6 en el ranking interior) o Información Comercial Española (7) abandonan las diez primeras posiciones cuando el objeto de estudio es su impacto internacional.

\section{UNA COMPARACIÓN CON LAS PRINCIPALES REVISTAS ALEMANAS, ITALIANAS Y FRANCESAS}

En este apartado, finalmente, se ofrece una primera comparación de la visibilidad alcanzada por las revistas españolas en relación a las principales cabeceras 
alemanas, italianas y francesas ${ }^{5}$. El objeto del mismo es doble. Por una parte se trata de analizar qué papel desempeñan las revistas españolas en comparación con sus homólogas de algunos países con tradición académica en Economía y con lenguas oficiales distintas al inglés. En segundo lugar, el ejercicio se utiliza para identificar cuáles son las características de las cabeceras nacionales con mayor visibilidad internacional.

Para la realización de este ejercicio se parte de un trabajo previo en el que, por una parte, se ha seleccionado el conjunto de revistas objeto de estudio para cada país analizado. Por otra, al igual que para el caso español, se han contabilizado las citas recibidas por cada artículo publicado en cada una de estas revistas los años 1995-1999 desde su publicación hasta 2004.

Por lo que respecta a la selección de cabeceras, para el caso italiano, la lista de publicaciones nacionales considerada se ha extraído de la ofrecida por el Osservatorio della Recerca de la Università di Bologna. En particular, del total de revistas que este organismo considera en su relación de «publicaciones sin Factor de Impacto en Economía» se han considerado aquellas editadas en Italia a las que se les concede la tipología B1 o C1. Estas tipologías, según señala el informe, corresponden a revistas medias de difusión internacional con «referee» (B1) o a revistas menores con difusión internacional con «referee» $(\mathrm{C} 1)^{6}$. Resultado de esta selección es un listado de 25 revistas.

Para el estudio de las citas recibidas por las publicaciones editadas en Francia se ha contado con el estudio realizado por la Section 37 du Comité National «Economie et Societe» del Comité National de la Recherche Scientifique, bajo el título Classement des revues à comité de lecture en économie et en gestion ${ }^{7}$. El estudio establece una jerarquización de las revistas a partir de cinco niveles cuando se trata de revistas calificadas de generalistas y de cuatro cuando se consideran áreas específicas de especialización. Para el análisis del caso francés se han considerado todas las cabeceras editadas en francés de las revistas pertenecientes al listado de generalistas y aquéllas que, en las mismas condiciones, se incluyen en los niveles 1, 2 ó 3 de los listados por áreas de especialización ${ }^{8}$. Resultado de esta selección es un listado de 17 revistas.

${ }^{5}$ El detalle de la elaboración de los datos que se ofrecen en este apartado se puede encontrar en Pons y Tirado (2005).

${ }^{6} \mathrm{El}$ informe puede ser consultado en http://ossric.cineca.it//. La consulta fue realizada el $10 / 05 / 05$.

7 Cabría señalar que, atendiendo a la voluntad de cubrir la difusión de las revistas publicadas en francés, el estudio incluye el análisis de alguna cabecera editada en Bélgica.

${ }^{8}$ El nivel 1 corresponde a las revistas consideradas como «Revues d'excellence unanimement reconnues dans le champ». El 2 a «Revues de très haute qualité mais moins diffusées». Y el 3 a «Revues internationales de référence dans le champ». 
El caso alemán ha recibido un tratamiento distinto. Como en este caso no se han encontrado trabajos que permitieran realizar una selección en base a los mismos, el listado de las nueve cabeceras analizadas ha sido elaborado por un experto al que se le solicitó su realización ${ }^{9}$. Por lo que respecta al caso español se han considerado las 30 revistas que componen la base de datos explotada en los puntos previos.

Por tanto, se ha trabajado sobre las citas ISI recibidas por un conjunto de 81 cabeceras de Economía alemanas, españolas, francesas e italianas. De ellas se han cuantificado las recibidas por cada artículo publicado en este conjunto de revistas los años 1995-1999, y desde su publicación hasta 2004.

A continuación se ofrece un cuadro resumen (cuadro 7) en el que se presentan de forma ordenada las 15 revistas receptoras de un mayor número de citas, así como algunas características de las mismas.

La comparación internacional permite destacar el comparativamente elevado nivel de visibilidad alcanzado por las revistas españolas, que ocupan seis de las 15 primeras posiciones del ranking internacional. En el lado opuesto se situarían las publicaciones italianas, que no ocupan ninguna de estas plazas. La primera de las

CUADRO 7

CITAS ISI RECIBIDAS POR LOS ARTÍCULOS PUBLICADOS ENTRE 1995 Y 1999 EN EL QUINQUENIO POSTERIOR A SU PUBLICACIÓN

\begin{tabular}{|c|c|c|c|c|}
\hline & $\begin{array}{l}\text { Total } \\
\text { citas }\end{array}$ & Autocitas & $\begin{array}{c}\text { \%Filiación } \\
\text { País }\end{array}$ & Inglés \\
\hline 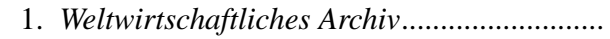 & 613 & 13,05 & 14,13 & $\mathrm{~S}$ \\
\hline 2. Annales d'Economie et Statistiques .. & 322 & 18,01 & 19,23 & $\mathrm{~S}$ \\
\hline 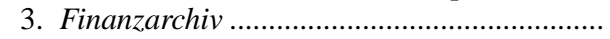 & 195 & 17,44 & 52,94 & $\mathrm{~S}$ \\
\hline 4. Revue d'Economie Industrielle.................. & 157 & 8,39 & 67,11 & $\mathrm{~S}$ \\
\hline 5. Investigaciones Económicas.......... & 110 & 19,82 & 70,39 & $\mathrm{~S}$ \\
\hline 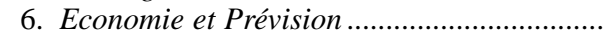 & 108 & 27,62 & 84,00 & $\mathrm{~N}$ \\
\hline 7. German Economic Review $*$........................ & 103 & 29,13 & 41,00 & $\mathrm{~S}$ \\
\hline 8. Papeles de Economía Española. & 83 & 4,82 & 84,34 & $\mathrm{~N}$ \\
\hline 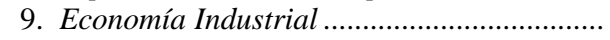 & 78 & 5,13 & 74,36 & $\mathrm{~N}$ \\
\hline 10. Revista Española de Economía/Spanish & & & & \\
\hline 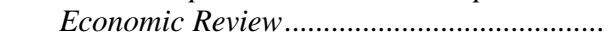 & 68 & 16,18 & 48,53 & $\mathrm{~S}$ \\
\hline 11. Revue d'Economie Politique...................... & 68 & 33,82 & 58,82 & $\mathrm{~S}$ \\
\hline 12. Revue d'Economie du Développement ........ & 64 & 31,25 & 82,26 & $\mathrm{~S}$ \\
\hline 13. Información Comercial Española ................ & 48 & 16,67 & 83,33 & $\mathrm{~N}$ \\
\hline 14. Revista de Historia Económica .................. & 45 & 13,33 & 57,78 & $\mathrm{~S}$ \\
\hline 15. Schmollers Jahrbuch*.................. & 44 & 13,64 & 65,91 & $\mathrm{~S}$ \\
\hline
\end{tabular}

Fuente: Elaboración propia.

${ }^{9}$ El listado que se presenta para el caso alemán es el sugerido por el Prof. Albrecht Ritschl, de la Humboldt University. 
cabeceras itialianas por número de citas, Rivista Internazionale di Scienze Economiche e Commerciali, ocupa el puesto 16 de esta clasificación.

Sin embargo, los primeros puestos de la clasificación son ocupados por dos revistas alemanas (puestos 1 y 3 ) y dos francesas ( 2 y 4). Además, el liderato de estas publicaciones, sobre todo por lo que respecta a las dos primeras, es muy nítido. La primera cabecera, la alemana Weltwirtschaftliches Archiv, recibe un número de citas que prácticamente sextuplica al de la mejor revista española (Investigaciones Económicas, situada en quinto lugar de la clasificación. El número de citas recibido por la segunda clasificada, la francesa Annales d'Economie et Statistiques, casi triplica al de la citada cabecera española.

En este sentido, parece razonable preguntarse qué elementos caracterizan a las revistas que reciben un mayor número de citas internacionales, y en particular a aquéllas que ocupan las posiciones más destacadas de esta clasificación. Las columnas 3 (Autocitas), 4 (\% Filiación País) y 5 (Inglés) ofrecen información que puede ayudar a dar respuesta a esta cuestión.

En primer lugar, se observa cómo todas estas revistas tienen una dependencia muy baja de las autocitas. De hecho, el número de citas recibidas prácticamente no guarda relación con las publicaciones realizadas por los autores de los artículos citados. Sin embargo, con la excepción de las cabeceras que encabezan la clasificación, sigue existiendo una clara relación entre la filiación por países de los autores de los artículos citantes y las citas recibidas por las cabeceras de cada país.

Por lo que respecta al idioma en el que se realiza la publicación, la columna 5 nos muestra cómo las revistas que reciben un mayor número de citas bien son publicadas íntegramente en inglés, bien publican en este idioma una parte relevante de la totalidad de aportaciones. De hecho, 11 de las 15 revistas analizadas así lo hacen, entre ellas las cinco mejor clasificadas. Las dos revistas que, con cierta distancia, encabezan esta clasificación se publican íntegramente en inglés.

En resumen, las revistas españolas ocupan una posición aceptable por lo que respecta a su visibilidad internacional. No obstante, la mejor de las cabeceras españolas se encuentra aún a cierta distancia de las cabeceras alemanas o francesas más citadas. Éstas muestran una más marcada internacionalización de su oferta que se cimenta en la publicación en inglés de sus originales y que les permite desvincular un volumen importante de citas recibidas de las publicaciones realizadas por autores con filiación en universidades o centros de investigación del mismo país que la revista citada.

\section{CONSIDERACIONES FINALES}

En este trabajo se ha realizado un primer análisis del impacto que tienen los artículos publicados en las revistas españolas de economía en el ámbito interna- 
cional. Se entiende que éste es un paso obligado en la mejora de la evaluación de la actividad investigadora realizada por los individuos o las instituciones de investigación en España que, a la vez, complementa los esfuerzos ya realizados acerca de la delimitación del impacto de las publicaciones españolas de economía en el ámbito interior.

Para su realización se ha partido del análisis de las más de 2.000 citas que componen la totalidad de las recibidas por los artículos publicados en las 30 revistas españolas de economía con mayor índice de impacto a escala interior en todas las revistas que componen las bases de datos del ISI a lo largo de su historia. El trabajo de base realizado, además, ha permitido destacar algunas características propias tanto de las citas (qué se cita, quién cita, a qué institución pertenece) como de las revistas citantes (cuáles son, cuál es su factor de impacto en el SSCI).

Esta primera aproximación, al margen de servir para mostrar cómo las revistas españolas de economía con mayor trayectoria histórica han sido las más citadas, ha permitido mostrar cómo, en líneas generales, las citas recibidas por los artículos españoles están asociadas a la presencia de artículos elaborados por autores españoles en las revistas internacionales y que, con toda probabilidad por este motivo, suelen aparecen en revistas ISI de un factor de impacto medio-bajo. No obstante, también ha permitido señalar que el volumen de citas recibidas no debe considerarse irrelevante.

Partiendo de esta base de datos, se ha pasado a acotar temporalmente la muestra objeto de análisis, de forma que se evitara favorecer la posición de las revistas con mayor trayectoria histórica de publicación. En particular, el periodo analizado han sido los años 1995-1999, considerando las citas recibidas por un artículo en los años posteriores a su publicación. Es sobre esta muestra, que proporciona unas características por lo que respecta a la calidad de las citas similar a la general, sobre la que se han construido los factores e índices de impacto internacional.

En este sentido, cabe señalar que al ponderar el volumen bruto de citas por el número de artículos publicados durante el periodo objeto de análisis, revistas con un amplio volumen de citas se han visto superadas por cabeceras que reciben un volumen superior en proporción a la producción científica publicada. En particular, cabe destacar cómo tres de las cinco cabeceras que recibían un mayor volumen de citas brutas no se sitúan entre las cinco primeras. Papeles de Economía Española, Economía Industrial e Información Comercial Española abandonan este grupo y son reemplazadas por Moneda y Crédito, Revista de Historia Económica y Revista de Economía Aplicada. Además, los resultados son robustos a la utilización de alternativas de ponderación de la producción científica publicada por cada revista como el número de páginas estandarizadas.

En cualquier caso, el análisis de los valores calculados para los índices de impacto internacional de las revistas españolas de economía ha servido para mostrar tanto el bajo impacto medio de las mismas como la existencia de grandes dife- 
rencias entre las distintas publicaciones. En particular, dos revistas destacan claramente por encima de la media en cuanto a su impacto internacional: Revista Española de Economía e Investigaciones Económicas.

Estos resultados han sido comparados con los obtenidos en los estudios realizados sobre el impacto interior de las revistas españolas de economía. En este sentido, se ha observado cómo un grupo importante de revistas mantienen los primeros lugares del ranking con independencia de cuál sea el observatorio utilizado (mercado interior o internacional). No obstante, se ha identificado la existencia de algunas revistas, en la mayor parte de casos ligadas a algunas especialidades o áreas de investigación específicas, que se sitúan en niveles del ranking muy diversos en función de cuál sea el tipo de impacto que concentre nuestra atención, internacional o nacional. Por ejemplo, las revistas del área de Historia Económica se sitúan en mejor posición en el ranking de impacto internacional, las de Contabilidad, Finanzas, Economía Pública o Industrial en el nacional.

Por lo que respecta a la visibilidad de las revistas españolas en comparación con la alcanzada por publicaciones de carácter similar, pero editadas en Alemania, Italia o Francia, el trabajo realizado permite señalar que las revistas españolas ocupan una posición relevante en este conjunto. De hecho, seis de las 15 cabeceras más citadas son españolas. Sin embargo, la primera revista española aparece en la quinta posición de este ranking y a cierta distancia de las grandes publicaciones alemanes o francesas, que copan las primeras posiciones. Éstas, finalmente, muestran una más marcada internacionalización de su oferta.

A modo de hipótesis se puede apuntar que ésta se cimentaría, entre otros aspectos, en la publicación en inglés de sus originales, elemento que permite desvincular un volumen importante de citas recibidas de las publicaciones realizadas por autores con filiación en universidades o centros de investigación del mismo país que la revista citada.

\section{BIBLIOGRAFÍA}

Bergantiños, G.; Da Rocha, J. M., y Polomé, P. (2002): «La investigación española en economía, 1995-1999». Investigaciones Económicas 26, pp. 373-392.

Combes, P. P., y LinNemer, L. (2003): «Where are the economists who publish? Publication concentration and ránkings in Europe based on cumulative publications». Journal of the European Economic Association 1, pp. 1250-1308.

Conroy, M. E.; DusAnsky, R.; DrukKer, D., y KildegaARd, A. (1995): «The productivity of economics departments in the U.S.: Publications in the core journals». Journal of Economic Literature 33, pp. 1966-1971.

Coupé, T. (2003): «Revealed perfomances: Worldwide ránkings of economists and economics departments, 1990-2000». Journal of the European Economic Association 1, pp. 1309-1345. 
Dolado, J. J.; García-Romero, A., y Zamarro, G. (2003): «Publishing perfomance in economics: Spanish ránkings (1990-1999)». Spanish Economic Review 5, pp. 85-100.

DusAnsky, R., y Vernon, C. J. (1998): «Ránkings of U.S. economics departments». Journal of Economic Perspectives 12, pp. 151-170.

García, P.; Lafuente, A.; Montañés, A., y Sanz, F. (1999a): «Producción científica en economía según publicaciones en revistas internacionales: El caso español». Papeles de Economía Española 81, pp. 49-57.

García, P.; MontaÑÉs, A., y SANZ, F. (1999b): «La investigación en economía en España: Mercado nacional ¿versus? mercado internacional». Revista de Economía Aplicada 20, pp. 167-187.

Kalaitzidakis, P.; Mamuneas, T. P., y Stengos, T. (2003): «Rankings of academic journals and institutions in economics». Journal of the European Economic Association 1, pp. 1346-1366.

Pons, J., y TiRADO, D. A. (1999): «¿Quién publica en las revistas españolas de economía?». Revista de Economía Aplicada 20, pp. 139-166.

- (2001): «El impacto de las revistas españolas de economía. Un estudio referido a 1998». Información Comercial Española. Revista de Economía 795, pp. 179-188.

- (2005): «Is There Life Beyond the ISI Journals lists? The International Impact of Spanish, Italian, French and German Economics Journals». Working Papers Series, 0508. Madrid: Instituto Laureano Figuerola de Historia Económica, Universidad Carlos III de Madrid.

Pons, J.; TiRADO, D. A., y Román, C. (2006): «¿Quién publica en las revistas españolas de Economía? Seis años después». Revista de Economía Aplicada, en prensa.

ScotT, L. C., y Mitias, P. M. (1996): «Trends in rankings of economics departments in the U.S.: An update». Economic Inquiry 34, pp. 378-400.

TIRADO, D. A., y Pons, J. (2003): «Una primera aproximación al impacto de las revistas españolas de Historia Económica». Boletín de la Asociación Española de Historia Económica 27, pp. 21-24.

Tombazos, C. G. (2005): «A revisionist perspective of European research in Economics». European Economic Review 49, pp. 251-277.

VelázQUEZ, F. J. (2003): «El impacto de las revistas científicas de economía en España». Documento de Trabajo EEE-160. Madrid: FEDEA. Disponible en http://www.fedea.es/pub/eee/eee160.pdf.

- (2004): «Calidad y difusión de las revistas españolas de economía y empresa». Papeles de Economía Española 100 (2), pp. 276-292. 